\title{
Reprobación, síntoma de deserción escolar en licenciatura en Nutrición de la Universidad Autónoma del Carmen
}

\author{
Reprobation, Symptom of School Dropout in Nutrition Degree from the \\ Universidad Autónoma del Carmen
}

Fracasso, sintoma de abandono escolar em Bacharelado em Nutrição na Universidade Autônoma de Carmen

Ángel Esteban Torres-Zapata

Universidad Autónoma del Carmen, México

etorre@pampano.unacar.mx https://orcid.org/0000-0002-6066-3258

Javier Rivera Domínguez

Universidad Autónoma del Carmen, México jrdominguez@pampano.unacar.mx https://orcid.org/0000-0001-7660-4773

Patricia Flores López Universidad Autónoma del Carmen, México pflores@pampano.unacar.com https://orcid.org/0000-0002-9099-471X

María del Pilar García Reyes Universidad Autónoma del Carmen, México mpgarcía@pampano.unacar.mx https://orcid.org/0000-0002-1655-1857

Dariola Astrid Castillo Trejo Universidad Autónoma del Carmen, México dcastillo@pampano.unacar.mx https://orcid.org/0000-0002-6771-0168 


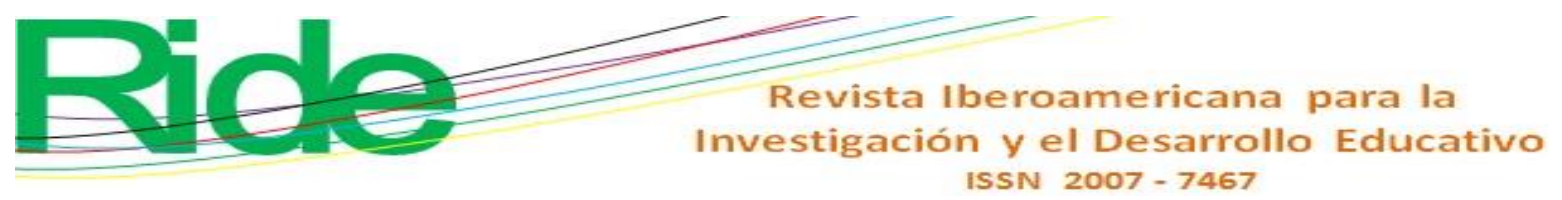

\section{Resumen}

La reprobación es la decisión de un profesor o jurado respecto al deficiente desempeño académico de un alumno, por lo tanto, no se le conceden los créditos correspondientes y debe repetir el curso o volver a realizar el examen. Las implicaciones de la reprobación son graves, ya que generan baja autoestima y se consideran como una variable asociada con la deserción escolar. El objetivo de esta investigación fue determinar la relación entre el índice de reprobación e índice de deserción escolar en los estudiantes de licenciatura en Nutrición de la Universidad Autónoma del Carmen. El presente estudio tiene un enfoque cuantitativo, de alcance exploratorio, descriptivo y diseño no experimental con una delineación transversal. La determinación del índice de reprobación y la tasa de deserción se obtuvo con estadística descriptiva y la correlación entre la reprobación y la deserción escolar se determinó con la prueba de Pearson. Se consideró la población total de 391 estudiantes que ingresó a dicho programa entre agosto-diciembre 2010 y agosto-diciembre 2017, con la que se determinó el índice de reprobación. De los resultados obtenidos se identificó que el índice de reprobación general encontrado fue de $24.24 \% \pm 12.68$ y con una media de apertura de cursos de $8.79 \pm 3.06$. El comportamiento identificado por los estudiantes ante la deserción mostró una media de $2.35 \pm 0.98$ ciclos avanzados, una media de $8.6 \pm 3.15$ cursos aprobados y una media $6.46 \pm 2.74$ de cursos reprobados al momento de desertar. La correlación entre el índice de desertores por cohorte generacional (2010 a 2017) y el índice de reprobación por cohorte generacional (2010 a 2017) en estudiantes de la licenciatura en Nutrición, a través del coeficiente de correlación de Pearson, fue de 0.939 y un valor de $p=0.001$. Asimismo, $96 \%$ de los estudiantes desertores en sus trayectorias escolares tienen entre $40 \%$ y $80 \%$ de cursos reprobados. Un fenómeno identificado en el presente estudio fue el comportamiento de un estudiante desertor con respecto a los ciclos promedios que avanza y el promedio de cursos aprobados y reprobados; se encontró una media de $2.35 \pm 0.98$ ciclos avanzados, una media de $8.6 \pm 3.15$ cursos aprobados y una media $6.46 \pm 2.74$ de cursos reprobados al momento de desertar. Las implicaciones de un alto índice de reprobación son graves y se pueden considerar como una variable asociada con la deserción escolar, lo cual puede suponer un problema de calidad educativa.

Palabras claves: abandono escolar, estudiantes universitarios, índice reprobación. 


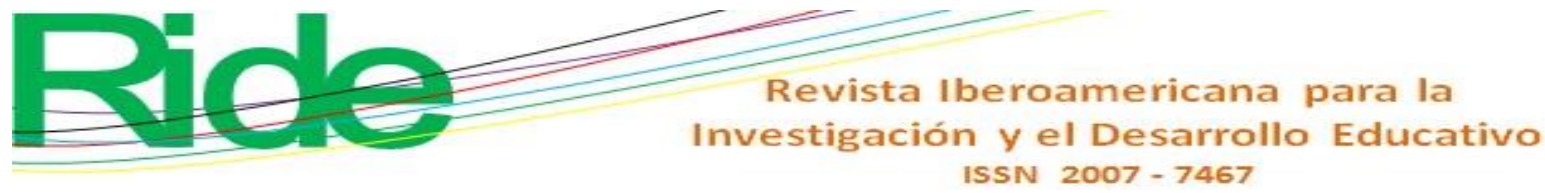

\section{Abstract}

Reprobation is defined as the judgement of a teacher or jury given to a student due to poor academic performance. Due to this situation, academic credits are not awarded to the students, so they must, in consequence, repeat a course or take another try on the test. Failure implications are dire, since they tend to build low self-esteem, and is closely related to school dropout. Determining the relationship between the reprobation and dropout rates of students at the Nutrition Bachelor's Degree Program (NBDP) at the Universidad Autónoma del Carmen is the main goal of this paper. This research has a non-experimental, quantitative, exploratory and descriptive approach, with a cross delineation. To determine reprobation and dropout rates descriptive statistics were applied; while Pearson's Chi-squared test was used to determine failure to dropout rates correlation. The totality of the 391 students enrolled in the NBDP between August 2010 to December 2017 were used to calculate the reprobation rate. The over-all reprobation rate found on the NBDP is $24.24 \%$ \pm 12.68 and an $8.79 \pm 3.06$ opened courses arithmetic mean. The overall behavior regarding academic advance on dropout students presented an arithmetic mean of $2.35 \pm 0.98$ academic cycles, they also presented an arithmetic mean of $8.6 \pm 3.15$ of courses approved and an arithmetic mean of $6.46 \pm 2.74$ of courses failed at the point where they dropped school. The correlation among dropout rates and reprobation rates for the NBDP 2010 - 2017 generational cohorts using the Pearson's Chi squared test was of 0.939 with a 0.001 value for $p$. Also, $96 \%$ of school dropouts has a course reprobation rate between $40 \%$ to $80 \%$. This implies that reprobation rates are dire since they are directly related to school dropout, which can also evidence a problem in education quality.

Keywords: dropping out, university students, reprobation.

\section{Resumo}

O fracasso é a decisão de um professor ou júri em relação ao fraco desempenho acadêmico de um aluno; portanto, os créditos correspondentes não são concedidos e devem repetir o curso ou refazer o exame. As implicações do fracasso são graves, pois geram baixa autoestima e são consideradas uma variável associada ao abandono escolar. O objetivo desta pesquisa foi determinar a relação entre a taxa de reprovação e a taxa de abandono em estudantes de graduação em Nutrição da Universidade Autônoma de Carmen. O presente estudo possui abordagem quantitativa, exploratória, descritiva e não experimental, com delineamento transversal. A determinação da taxa de reprovação e do abandono foi obtida com estatística descritiva e a correlação entre insucesso e

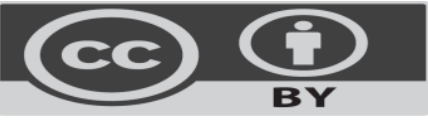




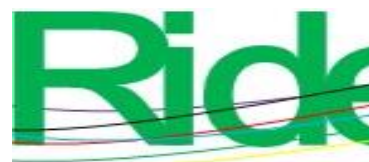

Revista Iberoamericana para la Investigación y el Desarrollo Educativo

ISSN $2007-7467$

abandono escolar foi determinada com o teste de Pearson. Foi considerada a população total de 391 estudantes que ingressaram no programa entre agosto e dezembro de 2010 e agosto e dezembro de 2017, com os quais foi determinada a taxa de reprovação. A partir dos resultados obtidos, identificou-se que o índice geral de reprovação encontrado foi de 24,24\% $\pm 12,68$ e com uma abertura média de curso de 8,79 $\pm 3,06$. O comportamento identificado pelos alunos antes do abandono mostrou uma média de 2,35 \pm 0,98 ciclos avançados, uma média de 8,6 \pm 3,15 cursos aprovados e uma média de 6,46 $\pm 2,74$ de cursos reprovados no momento do abandono. A correlação entre a taxa de evasão por coorte geracional (2010 a 2017) e a taxa de reprovação por coorte geracional (2010 a 2017) em estudantes de graduação em Nutrição, através do coeficiente de correlação de Pearson, foi de 0,939 e valor $\mathrm{p}=0,001$. Da mesma forma, $96 \%$ dos alunos que abandonam a escola em suas trajetórias escolares têm entre $40 \%$ e $80 \%$ dos cursos reprovados. Um fenômeno identificado no presente estudo foi o comportamento de um aluno que abandonou a escola em relação aos ciclos médios que estão avançando e à média de cursos aprovados e reprovados; foram encontrados uma média de 2,35 \pm 0,98 ciclos avançados, uma média de 8,6 \pm 3,15 cursos aprovados e uma média de 6,46 $\pm 2,74$ dos cursos reprovados no momento da deserção. As implicações de uma alta taxa de reprovação são graves e podem ser consideradas como uma variável associada ao abandono escolar, o que pode ser um problema de qualidade educacional.

Palavras-chave: evasão, estudantes universitários, taxa de reprovação.

Fecha Recepción: Agosto 2019

Fecha Aceptación: Enero 2020

\section{Introducción}

El término deserción escolar se ha definido como el abandono de las actividades escolares antes de terminar algún grado o nivel educativo (Torres, Acuña, Guadarrama, Solís y Flores, 2017). Este fenómeno es controversial dentro de las instituciones de educación superior, dado que no existe una causa específica a la que se le pueda atribuir, por ser una conducta multifactorial (Fallis y Opotow, 2003). El Reporte de la Encuesta Nacional de la Deserción en la Educación Media Superior (Secretaría de Educación Pública [SEP]-Consejo para la Evaluación de la Educación del Tipo Medio Superior [Copeems], 2012) indica que las principales causas de deserción escolar son: 1) escasa conexión entre la escuela y los intereses del estudiante, 2) insuficiente participación de los padres, 3) razones personales (necesidad de contar con un trabajo y obtener dinero; convertirse en madre o padre; estar a cargo del cuidado de algún familiar, entre otras) y 4) reprobación y falta de capacidad para superar los retos académicos. Esta última causa la confirman Sánchez (2015),

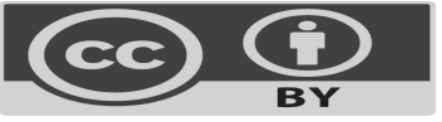




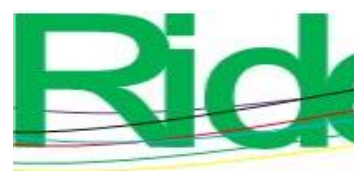

Revista Iberoamericana para la Investigación y el Desarrollo Educativo

ISSN $2007-7467$

Mendoza (2016) y Castellanos, Alvarado y Villamil (2018), un factor que conduce en muchas ocasiones al abandono de la escuela debido a la autopercepción del alumno del fracaso y a la conceptualización de que su bajo rendimiento no le permitirá continuar en los siguientes periodos.

La reprobación es la decisión de un profesor o jurado respecto al deficiente desempeño académico y del trabajo escolar de un alumno (curso o examen), por lo tanto, no se le conceden los créditos correspondientes y debe repetir el curso o volver a realizar el examen (Sánchez, 2015). Dicho de otra manera, los estudiantes reprobados son aquella parte de la población escolar sujeta a un programa curricular, pero que, por no haber cumplido con los requisitos académicos exigidos en el plan de estudios, no están en condiciones de ser promovidos al grado o nivel inmediato superior (Mendoza, 2016). De acuerdo con Piratoba y Barbosa (2013), las implicaciones de la reprobación son graves, ya que generan baja autoestima y se pueden considerar como una variable asociada con la deserción escolar.

Las escuelas de educación superior tienen un alto índice de reprobación, que pone de manifiesto un problema de calidad educativa. Lograr la productividad en la educación requiere del análisis de los procedimientos y de los factores que afectan el proceso enseñanza aprendizaje (Flores, Camacho y Ontiveros, 2013). El programa educativo de licenciatura en Nutrición (PELN) adscrito a la Facultad de Ciencias de la Salud (FCS) de la Universidad Autónoma del Carmen (Unacar) no está exento de este fenómeno, ya que desde que fue creado, en 2002, se ha presentado, sin generar estrategias de análisis y atención. Desde su creación a la fecha, al PELN se le han realizado tres actualizaciones, la última en el año 2010, alineada con la puesta en marcha del actual Modelo Educativo Acalán, que está centrado en el aprendizaje para toda la vida, con un enfoque por competencias, el cual ofrece a los estudiantes una formación integral y pertinente, con currículos flexibles y una filosofía de la educación para toda la vida. Sus principales características son:

- Se centra en el aprendizaje del estudiante;

- $\quad$ Promueve el aprendizaje significativo, y

- La organización curricular contempla tres tipos de competencias: 1) competencias genéricas, 2) competencias interdisciplinarias y 3) competencias específicas.

Las competencias genéricas constituyen la base común en la formación profesional a través de los programas educativos en la Unacar, y son sistémicas o integradoras, transversales y transferibles. Estas competencias son las siguientes: 1) cultura de salud, 2) comunicación y relación social, 3) cultura emprendedora, educación y transdisciplina, 4) universidad, ciencia y humanismo,

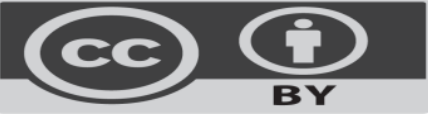




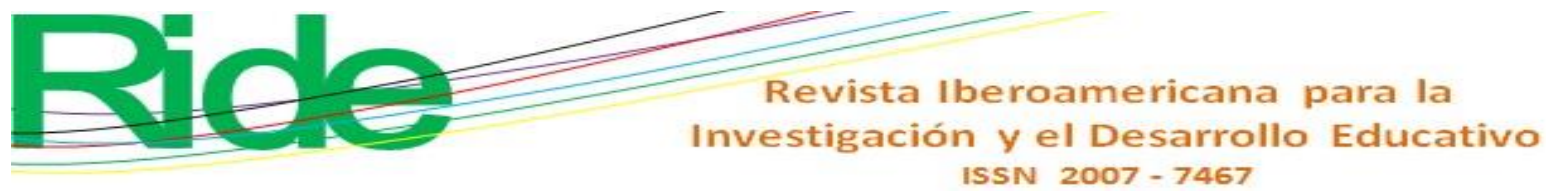

5) educación para la sustentabilidad y 6) dominio de las tecnologías de información y comunicación.

Respeto a los cursos que contribuyen a lograr las competencias genéricas, $82 \%$ se encuentran ubicados en los primeros cuatro ciclos de la carrera. Durante estos el estudiante del PELN se encuentra matriculado junto con alumnos de otro programa educativo de la Unacar.

Las competencias interdisciplinarias identifican la formación de los estudiantes de una facultad con base en las áreas disciplinares que forman los programas educativos. Sus características son similares a las genéricas. La FCS establece como estas las puestas a continuación: 1) promoción de la salud y 2) responsabilidad social en salud; competencias que identificarán todas las acciones generales que un profesional en salud adquiere y desarrolla.

En los cursos que contribuyen a estas competencias, el estudiante del PELN toma la clase con estudiantes de los diversos programas educativos de la FCS (Enfermería, Psicología Clínica, Fisioterapia, Medicina y Educación Física y Deporte).

Las competencias específicas son las propias de la profesión, con las que se prepara al estudiante para un desempeño laboral o profesional específico. El PELN incluye entre este tipo de competencias las puestas aquí: 1) valoración y diagnóstico nutricional integral individual y colectiva, 2) intervención nutricional y alimentaria, 3) orientación nutricional, 4) administración de servicios de alimentación y 5) aprovechamiento integral y procesamiento de alimentos. Lo anterior permite alcanzar el perfil de egreso del licenciado en Nutrición al abordar las áreas de desempeño que la Asociación Mexicana de Miembros de Facultades y Escuelas de Nutrición (Ammfen) establece. Para lograr las competencias específicas, el PELN clasificó los cursos que contribuyen al desarrollo de estas en 1) básicas de la carrera, 2) profesionalizantes, 3) optativas y 4) terminales.

El PELN fue evaluado por primera vez por parte de los Comités Interinstitucionales para la Evaluación de la Educación Superior (Ciees) en 2010, y obtuvo el Nivel 1 con vigencia de cinco años; en 2016 recibió la visita para la evaluación de seguimiento y se le otorgó el mismo nivel por tres años más. A su vez, en 2016, alcanzó el estatus de Programa Educativo Acreditado por un periodo de cinco años por el Consejo Nacional para la Calidad de Programas Educativos en Nutriología (Concapren). Como resultado de estos procesos de evaluación externa, se han generado recomendaciones al PELN para el aseguramiento de la calidad educativa. Así, se observó como área de oportunidad en ambos ejercicios el tema de la trayectoria académica, específicamente la deserción (Torres et al., 2017) y la reprobación. Una de estas recomendaciones es caracterizar e 


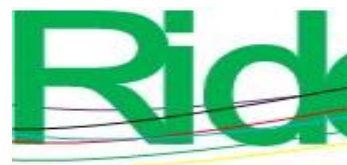

Revista Iberoamericana para la

Investigación y el Desarrollo Educativo

ISSN $2007-7467$

implementar estrategias para reducir los índices de reprobación y, por ende, disminuir la deserción escolar.

Por lo antes mencionado, este trabajo tiene los siguientes objetivos: 1) determinar el índice de reprobación, 2) determinar el índice de deserción y 3) analizar si existe relación entre el índice de reprobación e índice de deserción escolar en los estudiantes del PELN de la Unacar, desde el periodo agosto-diciembre 2010 a agosto-diciembre 2017, para contar con más información que permita obtener indicadores que sustenten el plan estratégico de actualización del programa educativo.

\section{Metodología}

Previo a iniciar la presente investigación, se solicitó autorización por escrito al gestor (coordinador) del PELN de la FCS de la Unacar, mencionando el objetivo general y los propósitos particulares del estudio. Posteriormente, se sometió a valoración por el comité de bioética, donde se descartaron conflictos de interés y se obtuvo un dictamen positivo. El estudio fue coordinado por el grupo disciplinar “Nutrición, Educación y Administración” del PELN de la FCS.

\section{Diseño de investigación}

El presente estudio tiene un enfoque cuantitativo, alcance exploratorio, descriptivo y diseño no experimental con una delineación transversal. Los propósitos principales fueron: 1) determinar el índice de reprobación escolar por cada competencia declaradas en la organización curricular, 2) determinar la tasa de deserción escolar en el PELN, 3) determinar ciclo promedio que avanzan los estudiantes desertores, promedio de aprobación y reprobación de cursos y 4) determinar si la reprobación escolar es un síntoma de la deserción escolar.

\section{Población}

Para obtener el índice de reprobación escolar, la población se constituyó con 391 estudiantes inscritos al PELN desde el periodo escolar agosto-diciembre 2010 al periodo agosto-diciembre 2017.

Para determinar ciclos promedios, aprobación, reprobación de cursos promedios y la relación entre la reprobación y deserción se tomó como población a la totalidad de 104 estudiantes con baja definitiva. 


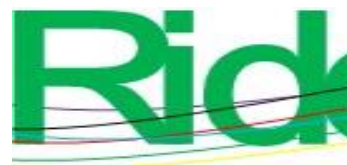

de

\section{Revista Iberoamericana para la \\ Investigación y el Desarrollo Educativo \\ ISSN $2007-7467$}

Fuentes para la obtención y/o recolección de la información

Los datos utilizados en esta investigación fueron suministrados por los sistemas informáticos institucionales Sistema Universitario de Control Escolar (SUCE) y Portal Ejecutivo, ambos con acceso del gestor del PELN.

\section{Instrumento de investigación y análisis estadístico}

Se analizaron los registros exportados de los sistemas institucionales a una base de datos en Excel. La información recolectada fue procesada y analizada con el apoyo del software Statistical Package for the Social Sciences versión 20. Para determinar el índice de reprobación y la tasa de deserción se presentó en porcentaje, frecuencias, medias, desviación estándar, valores máximos y mínimos; para determinar la correlación entre la reprobación y la deserción escolar se aplicó la prueba de Pearson.

\section{Resultados}

\section{Determinación del índice de reprobación}

Para la determinación del índice de reprobación, se tomó la totalidad de 391 estudiantes que ingresaron al PELN del periodo agosto-diciembre 2010 a agosto-diciembre 2017. El índice de reprobación general encontrado en el PELN es de $24.24 \% \pm 12.68$ y una media de apertura de cursos de $8.79 \pm 3.06$.

En la tabla 1 se muestra el índice de reprobación en relación con los cursos que contribuyen a las competencias genéricas, con una media $25.69 \% \pm 12.24$ de reprobación y una media de apertura de los cursos $11.16 \pm 2.88$. 


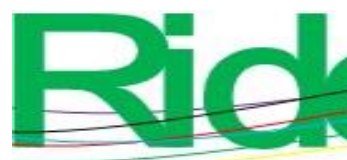

Revista Iberoamericana para la Investigación y el Desarrollo Educativo

ISSN $2007-7467$

Tabla 1. Índice de reprobación: cursos que contribuyen a las competencias genéricas

\begin{tabular}{|c|c|c|c|c|c|c|c|c|c|}
\hline \multirow[t]{2}{*}{ Cursos } & \multicolumn{5}{|c|}{ Inscritos } & \multicolumn{2}{|c|}{$\begin{array}{l}\text { Aprobación } \\
\text { de cursos }\end{array}$} & \multicolumn{2}{|c|}{$\begin{array}{l}\text { Reprobación } \\
\text { de cursos }\end{array}$} \\
\hline & NVCA* & $f$ & $\overline{\mathrm{X}}$ & Máx. & Mín. & $\mathrm{f}$ & $\%$ & $\mathrm{f}$ & $\%$ \\
\hline $\begin{array}{l}\text { Razonamiento } \\
\text { lógico }\end{array}$ & 13 & 334 & $25.69 \pm 19.47$ & 56 & 3 & 191 & 57.19 & 143 & 42.81 \\
\hline TMI** & 15 & 306 & $20.40 \pm 18.56$ & 52 & 1 & 207 & 67.65 & 99 & 32.35 \\
\hline $\mathrm{TCOE}^{* * *}$ & 14 & 403 & $28.79 \pm 21.68$ & 59 & 2 & 345 & 85.61 & 58 & 14.39 \\
\hline $\begin{array}{l}\text { Desarrollo } \\
\text { sustentable }\end{array}$ & 14 & 411 & $29.36 \pm 23.92$ & 63 & 1 & 325 & 79.08 & 86 & 20.92 \\
\hline Inglés I & 13 & 192 & $14.77 \pm 6.60$ & 32 & 5 & 103 & 53.65 & 89 & 46.35 \\
\hline Inglés II & 12 & 112 & $9.33 \pm 6.02$ & 22 & 2 & 65 & 58.04 & 47 & 41.96 \\
\hline Inglés III & 11 & 93 & $8.45 \pm 3.84$ & 16 & 1 & 70 & 75.27 & 23 & 24.73 \\
\hline Inglés IV & 10 & 51 & $5.10 \pm 2.02$ & 10 & 2 & 43 & 84.31 & 8 & 15.69 \\
\hline Emprendedores & 9 & 154 & $17.11 \pm 11.13$ & 36 & 1 & 130 & 84.42 & 24 & 15.58 \\
\hline $\mathrm{TE}^{* * * *}$ & 5 & 9 & $1.80 \pm 0.74$ & 3 & 1 & 7 & 77.78 & 2 & 22.22 \\
\hline TFTI***** & 11 & 132 & $12.00 \pm 8.14$ & 34 & 3 & 98 & 74.24 & 34 & 25.76 \\
\hline $\begin{array}{l}\text { Prácticas } \\
\text { profesionales }\end{array}$ & 7 & 90 & $12.86 \pm 12.86$ & 28 & 2 & 85 & 94.44 & 5 & 5.56 \\
\hline
\end{tabular}

*Número de veces que el curso se apertura.

**Tecnología y manejo de la información.

***Taller de comunicación oral y escrita.

$* * * *$ Taller de emprendedores.

*****Taller de formación temprana de investigadores.

Fuente: Elaboración propia

En la tabla 2 se muestra el índice de reprobación en relación con los cursos que contribuyen a las competencias interdisciplinarias; se determinó una media $31.28 \pm 13.38$, con una media de apertura de $11.78 \pm 1.97$. 


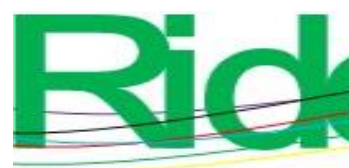

\section{Revista Iberoamericana para la Investigación y el Desarrollo Educativo \\ ISSN $2007-7467$}

Tabla 2. Índice de reprobación: cursos que contribuyen a las competencias interdisciplinarias

\begin{tabular}{|c|c|c|c|c|c|c|c|c|c|}
\hline \multirow[t]{2}{*}{ Cursos } & \multicolumn{5}{|c|}{ Inscritos } & \multicolumn{2}{|c|}{$\begin{array}{l}\text { Aprobación } \\
\text { de cursos }\end{array}$} & \multicolumn{2}{|c|}{$\begin{array}{l}\text { Reprobación } \\
\text { de cursos }\end{array}$} \\
\hline & NVCA* & $\mathrm{f}$ & $\bar{x}$ y D.E. & Máx. & Mín. & $\mathrm{f}$ & $\%$ & $\mathrm{f}$ & $\%$ \\
\hline Actividad física & 10 & 404 & $\begin{array}{c}40.40 \pm \\
19.61\end{array}$ & 62 & 4 & 350 & 86.63 & 54 & 13.37 \\
\hline $\begin{array}{l}\text { Educación } \\
\text { promoción } \\
\text { de la salud }\end{array}$ & 8 & 274 & $\begin{array}{c}34.25 \pm \\
13.97\end{array}$ & 48 & 3 & 225 & 82.12 & 49 & 17.88 \\
\hline Socioantropología & 13 & 403 & $\begin{array}{c}31.00 \pm \\
23.25\end{array}$ & 62 & 1 & 328 & 81.39 & 75 & 18.61 \\
\hline $\begin{array}{l}\text { Anatomía y } \\
\text { fisiología II }\end{array}$ & 11 & 302 & $\begin{array}{c}27.45 \pm \\
16.13\end{array}$ & 49 & 1 & 189 & 62.58 & 113 & 37.42 \\
\hline Bioquímica básica & 14 & 332 & $\begin{array}{c}23.71 \pm \\
17.64\end{array}$ & 53 & 4 & 205 & 61.75 & 127 & 38.25 \\
\hline $\begin{array}{l}\text { Anatomía y } \\
\text { fisiología I }\end{array}$ & 13 & 467 & $\begin{array}{c}35.92 \pm \\
21.09\end{array}$ & 60 & 3 & 267 & 57.17 & 200 & 42.83 \\
\hline Bioestadística básica & 13 & 245 & $\begin{array}{c}18.85 \pm \\
12.96\end{array}$ & 49 & 3 & 121 & 49.39 & 124 & 50.61 \\
\hline
\end{tabular}

*Número de veces que el curso se apertura.

Fuente: Elaboración propia

En las tablas 3, 4, 5 y 6 se muestran los índices de reprobación de los cursos que contribuyen a la generación de las competencias específicas, donde se encontró un índice de reprobación de $22.02 \pm 11.99$, con una media de apertura de cursos de $6.96 \pm 1.46$.

En la tabla 3 se muestra solo el comportamiento de los cursos que contribuyen a las competencias específicas del área básica de la carrera, teniendo una media de reprobación de 30.77 \pm 10.04 , con una media de apertura de cursos de $7.83 \pm 1.21$. 


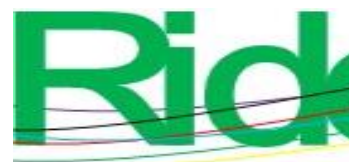

Revista Iberoamericana para la Investigación y el Desarrollo Educativo

ISSN $2007-7467$

Tabla 3. Índice de reprobación: cursos que contribuyen a las competencias específicas. Básicas de la carrera

\begin{tabular}{|c|c|c|c|c|c|c|c|c|c|c|}
\hline \multirow[t]{2}{*}{ Cursos } & \multicolumn{6}{|c|}{ Inscritos } & \multicolumn{2}{|c|}{$\begin{array}{c}\text { Aprobaci } \\
\text { ón } \\
\text { de cursos }\end{array}$} & \multicolumn{2}{|c|}{$\begin{array}{l}\text { Reprobac } \\
\text { ión } \\
\text { de cursos }\end{array}$} \\
\hline & $\begin{array}{c}\text { NVC } \\
\text { A* }\end{array}$ & $\mathrm{N}$ & $\overline{\mathrm{X}}$ & \pm & $\begin{array}{c}\text { Má } \\
\text { X. }\end{array}$ & Mín. & $\mathrm{N}$ & $\%$ & $\mathrm{~N}$ & $\%$ \\
\hline Nutrición básica & 10 & 319 & $\begin{array}{c}31.9 \\
0\end{array}$ & $\begin{array}{c}14.9 \\
4\end{array}$ & 48 & 4 & $\begin{array}{l}2 \\
6 \\
6\end{array}$ & 83.39 & 53 & $\begin{array}{l}16 . \\
61\end{array}$ \\
\hline Administración general & 8 & 251 & $\begin{array}{c}31.3 \\
8\end{array}$ & $\begin{array}{c}10.1 \\
3\end{array}$ & 41 & 14 & $\begin{array}{l}2 \\
0 \\
8\end{array}$ & 82.87 & 43 & $\begin{array}{l}17 . \\
13\end{array}$ \\
\hline Nutrición y metabolismo & 8 & 273 & $\begin{array}{c}34.1 \\
3\end{array}$ & 8.83 & 43 & 21 & $\begin{array}{l}1 \\
7 \\
7\end{array}$ & 64.84 & 96 & $\begin{array}{l}35 . \\
16\end{array}$ \\
\hline Taller de cálculo & 7 & 277 & $\begin{array}{c}39.5 \\
7\end{array}$ & 6.98 & 49 & 26 & $\begin{array}{l}1 \\
7 \\
5\end{array}$ & 63.18 & 102 & $\begin{array}{l}36 . \\
82\end{array}$ \\
\hline Epidemiología & 8 & 184 & $\begin{array}{c}23.0 \\
0\end{array}$ & $\begin{array}{c}16.0 \\
3\end{array}$ & 53 & 1 & $\begin{array}{l}1 \\
1 \\
6\end{array}$ & 63.04 & 68 & $\begin{array}{l}36 . \\
96\end{array}$ \\
\hline $\begin{array}{l}\text { Farmacología de la } \\
\text { nutrición }\end{array}$ & 6 & 224 & $\begin{array}{c}37.3 \\
3\end{array}$ & 7.93 & 52 & 27 & $\begin{array}{l}1 \\
3 \\
0\end{array}$ & 58.04 & 94 & $\begin{array}{l}41 . \\
96\end{array}$ \\
\hline
\end{tabular}

*Número de veces que el curso se apertura.

Fuente: Elaboración propia

En la tabla 4, por su parte, se muestra solo el comportamiento de los cursos que contribuyen a las competencias específicas del área profesionalizante de la carrera, teniendo una media de reprobación de $21.04 \pm 9.22$ con una media de apertura de cursos de $6.73 \pm 1.11$. 


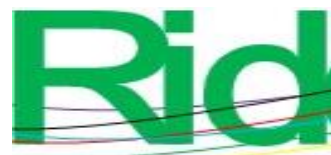

Revista Iberoamericana para la Investigación y el Desarrollo Educativo

ISSN $2007-7467$

Tabla 4. Índice de reprobación: cursos que contribuyen a las competencias específicas.

Profesionalizante

\begin{tabular}{|c|c|c|c|c|c|c|c|c|c|c|}
\hline \multirow[t]{2}{*}{ Cursos } & \multicolumn{6}{|c|}{ Inscritos } & \multicolumn{2}{|c|}{$\begin{array}{l}\text { Aprobación } \\
\text { de cursos }\end{array}$} & \multicolumn{2}{|c|}{$\begin{array}{c}\text { Reproba } \\
\text { ción } \\
\text { de cursos }\end{array}$} \\
\hline & $\begin{array}{l}\mathrm{NVC} \\
\mathrm{A}^{*}\end{array}$ & $\mathrm{f}$ & $\overline{\mathrm{x}}$ & $\mathrm{DE}$ & $\begin{array}{l}\text { Má } \\
\text { x. }\end{array}$ & $\begin{array}{c}\text { Mí } \\
\text { n. }\end{array}$ & $\mathrm{f}$ & $\%$ & f & $\%$ \\
\hline $\mathrm{ASA}^{* *}$ & 6 & $\begin{array}{l}2 \\
1 \\
3\end{array}$ & $\begin{array}{c}35.5 \\
0\end{array}$ & 2.87 & 39 & 6 & 168 & $\begin{array}{c}78.8 \\
7\end{array}$ & 45 & $\begin{array}{l}21 . \\
13\end{array}$ \\
\hline Alimentación y sociedad & 7 & $\begin{array}{l}2 \\
5 \\
1\end{array}$ & $\begin{array}{c}35.8 \\
6\end{array}$ & 9.44 & 51 & 25 & 212 & $\begin{array}{c}84.4 \\
6\end{array}$ & 39 & $\begin{array}{l}15 . \\
54\end{array}$ \\
\hline Arte culinario & 7 & $\begin{array}{l}2 \\
5 \\
2\end{array}$ & $\begin{array}{c}36.0 \\
0\end{array}$ & 7.67 & 45 & 23 & 216 & $\begin{array}{c}85.7 \\
1\end{array}$ & 36 & $\begin{array}{l}14 . \\
29\end{array}$ \\
\hline Ciencias de los alimentos & 6 & $\begin{array}{l}1 \\
8 \\
9\end{array}$ & $\begin{array}{c}31.5 \\
0\end{array}$ & 4.23 & 36 & 23 & 164 & $\begin{array}{c}86.7 \\
7\end{array}$ & 25 & $\begin{array}{l}13 . \\
23\end{array}$ \\
\hline Dietoterapia I & 7 & $\begin{array}{l}1 \\
4 \\
6\end{array}$ & $\begin{array}{c}20.8 \\
6\end{array}$ & $\begin{array}{c}10.9 \\
7\end{array}$ & 36 & 6 & 107 & $\begin{array}{c}73.2 \\
9\end{array}$ & 39 & $\begin{array}{l}26 . \\
71\end{array}$ \\
\hline Dietoterapia II & 7 & $\begin{array}{l}1 \\
2 \\
4\end{array}$ & $\begin{array}{c}17.7 \\
1\end{array}$ & 6.51 & 28 & 9 & 112 & $\begin{array}{c}90.3 \\
2\end{array}$ & 12 & $\begin{array}{c}9.6 \\
8\end{array}$ \\
\hline IAN*** & 5 & $\begin{array}{l}1 \\
5 \\
5\end{array}$ & $\begin{array}{c}31.0 \\
0\end{array}$ & 5.54 & 38 & 23 & 105 & $\begin{array}{c}67.7 \\
4\end{array}$ & 50 & $\begin{array}{l}32 . \\
26\end{array}$ \\
\hline MTA.**** & 10 & $\begin{array}{l}2 \\
9 \\
4\end{array}$ & $\begin{array}{c}29.4 \\
0\end{array}$ & $\begin{array}{c}13.7 \\
9\end{array}$ & 50 & 9 & 211 & $\begin{array}{c}71.7 \\
7\end{array}$ & 83 & $\begin{array}{l}28 . \\
23\end{array}$ \\
\hline
\end{tabular}




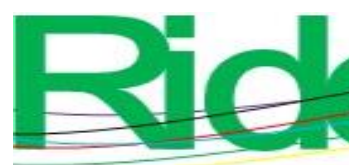

Revista Iberoamericana para la Investigación y el Desarrollo Educativo

ISSN 2007 - 7467

\begin{tabular}{|c|c|c|c|c|c|c|c|c|c|c|}
\hline Nutrición clínica I & 6 & $\begin{array}{l}1 \\
4 \\
3\end{array}$ & $\begin{array}{c}23.8 \\
3\end{array}$ & 9.08 & 34 & 11 & 90 & $\begin{array}{c}62.9 \\
4\end{array}$ & 53 & $\begin{array}{l}37 . \\
06\end{array}$ \\
\hline Nutrición clínica II & 8 & $\begin{array}{l}1 \\
3 \\
9\end{array}$ & $\begin{array}{c}17.3 \\
8\end{array}$ & 8.48 & 30 & 4 & 105 & $\begin{array}{c}75.5 \\
4\end{array}$ & 34 & $\begin{array}{r}24 . \\
46\end{array}$ \\
\hline Nutrición clínica pediátrica & 6 & $\begin{array}{l}1 \\
5 \\
0\end{array}$ & $\begin{array}{c}25.0 \\
0\end{array}$ & 8.28 & 36 & 10 & 99 & $\begin{array}{c}66.0 \\
0\end{array}$ & 51 & $\begin{array}{l}34 . \\
00\end{array}$ \\
\hline Nutrición comunitaria I & 7 & $\begin{array}{l}1 \\
7 \\
6\end{array}$ & $\begin{array}{c}25.1 \\
4\end{array}$ & $\begin{array}{c}13.6 \\
6\end{array}$ & 44 & 2 & 119 & $\begin{array}{c}67.6 \\
1\end{array}$ & 57 & $\begin{array}{l}32 . \\
39\end{array}$ \\
\hline Nutrición comunitaria II & 6 & $\begin{array}{l}8 \\
7\end{array}$ & $\begin{array}{c}14.5 \\
0\end{array}$ & 7.58 & 25 & 3 & 83 & $\begin{array}{c}95.4 \\
0\end{array}$ & 4 & $\begin{array}{c}4.6 \\
0\end{array}$ \\
\hline Nutrición en el individuo sano & 7 & $\begin{array}{l}1 \\
9 \\
9\end{array}$ & $\begin{array}{c}28.4 \\
3\end{array}$ & 8.05 & 38 & 16 & 160 & $\begin{array}{c}80.4 \\
0\end{array}$ & 39 & $\begin{array}{r}19 . \\
60\end{array}$ \\
\hline PLSA $* * * * *$ & 8 & $\begin{array}{l}3 \\
5 \\
5\end{array}$ & $\begin{array}{c}44.3 \\
8\end{array}$ & $\begin{array}{c}18.6 \\
1\end{array}$ & 9 & 8 & 282 & $\begin{array}{c}79.4 \\
4\end{array}$ & 73 & $\begin{array}{r}20 . \\
56\end{array}$ \\
\hline Psicología de la nutrición & 7 & $\begin{array}{l}1 \\
8 \\
5\end{array}$ & $\begin{array}{c}26.4 \\
3\end{array}$ & $\begin{array}{c}11.2 \\
4\end{array}$ & 37 & 1 & 165 & $\begin{array}{c}89.1 \\
9\end{array}$ & 20 & $\begin{array}{l}10 . \\
81\end{array}$ \\
\hline Seminario de tesis & 5 & $\begin{array}{l}1 \\
3 \\
6\end{array}$ & $\begin{array}{c}27.2 \\
0\end{array}$ & 2.99 & 31 & 22 & 110 & $\begin{array}{c}80.8 \\
8\end{array}$ & 26 & $\begin{array}{l}19 . \\
12\end{array}$ \\
\hline TEEN****** & 7 & $\begin{array}{l}2 \\
2 \\
5\end{array}$ & $\begin{array}{c}32.1 \\
4\end{array}$ & $\begin{array}{c}11.0 \\
3\end{array}$ & 42 & 6 & 163 & $\begin{array}{c}72.4 \\
4\end{array}$ & 62 & $\begin{array}{l}27 . \\
56\end{array}$ \\
\hline
\end{tabular}




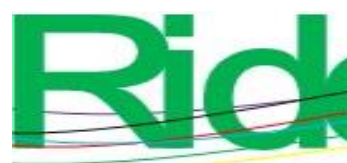

Revista Iberoamericana para la Investigación y el Desarrollo Educativo

ISSN $2007-7467$

\begin{tabular}{|l|c|c|c|c|c|c|c|c|c|c|}
\hline Tecnología de alimentos & 6 & 1 & 28.8 & 6.71 & 35 & 16 & 158 & 91.3 & 15 & 8.6 \\
& & 7 & 3 & & & & & 3 & & 7 \\
& & 3 & & & & & & & & \\
\hline
\end{tabular}

*Número de Veces que el Curso se apertura.

**Administración de servicios de alimentación.

***Investigación aplicada a la nutrición

****Microbiología y toxicología de alimentos.

******Producción, legislación y seguridad alimentaria.

******Taller de evaluación del estado nutricio.

Fuente: Elaboración propia

En la tabla 5 se muestra solo el comportamiento de los cursos que contribuyen a las competencias específicas optativas de la carrera, teniendo una media de reprobación de $34.06 \pm$ 9.58 .

Tabla 5. Índice de reprobación: cursos que contribuyen a las competencias específicas. Optativas

\begin{tabular}{|l|c|c|c|c|c|c|c|c|c|c|}
\hline Cursos & \multicolumn{5}{|c|}{ Inscritos } & \multicolumn{2}{c|}{$\begin{array}{c}\text { Aprobación } \\
\text { de cursos }\end{array}$} & \multicolumn{2}{c|}{$\begin{array}{c}\text { Reprobación } \\
\text { de cursos }\end{array}$} \\
\cline { 2 - 11 } & NVCA* & $\mathrm{f}$ & $\overline{\mathrm{x}}$ & \pm & MÁX. & MÍN. & $\mathrm{f}$ & $\%$ & $\mathrm{f}$ & $\%$ \\
\hline $\begin{array}{l}\text { Evaluación } \\
\text { sensorial de } \\
\text { los alimentos }\end{array}$ & 7 & 192 & 27.43 & 12.46 & 40 & 2 & 145 & 75.52 & 47 & 24.48 \\
\hline $\begin{array}{l}\text { Nutrición en el } \\
\text { deportista }\end{array}$ & 2 & 55 & 27.50 & 7.50 & 35 & 20 & 31 & 56.36 & 24 & 43.64 \\
\hline
\end{tabular}

*Número de veces que el curso se apertura.

Fuente: Elaboración propia

En la tabla 6, finalmente, se muestra solo el comportamiento de los cursos que contribuyen a las específicas terminales de la carrera, teniendo una media de reprobación de $2.68 \pm 2.34$. 


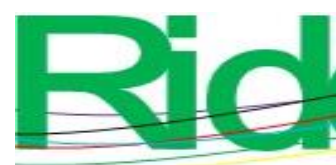

Revista Iberoamericana para la Investigación y el Desarrollo Educativo

ISSN $2007-7467$

Tabla 6. Índice de reprobación: cursos que contribuyen a las competencias específicas.

Terminales

\begin{tabular}{|c|c|c|c|c|c|c|c|c|c|c|}
\hline \multirow[t]{2}{*}{ Cursos } & \multicolumn{6}{|c|}{ Inscritos } & \multicolumn{2}{|c|}{$\begin{array}{c}\text { Aprobación } \\
\text { de cursos }\end{array}$} & \multicolumn{2}{|c|}{$\begin{array}{c}\text { Reprobación } \\
\text { de cursos }\end{array}$} \\
\hline & $\mathrm{NVCA}^{*}$ & $\mathrm{f}$ & $\overline{\mathrm{X}}$ & \pm & Máx. & Mín. & $\mathrm{f}$ & $\%$ & $\mathrm{f}$ & $\%$ \\
\hline $\begin{array}{l}\text { Prácticas } \\
\text { hospitalarias }\end{array}$ & 8 & 80 & 10.00 & 8.36 & 28 & 1 & 80 & 100 & 0 & 0.00 \\
\hline $\begin{array}{l}\text { Prácticas } \\
\text { comunitarias }\end{array}$ & 7 & 86 & 12.29 & 8.71 & 27 & 1 & 84 & 97.67 & 2 & 2.33 \\
\hline $\begin{array}{ll}\text { Prácticas } & \text { de } \\
\text { servicios } & \text { de } \\
\text { alimentación } & \end{array}$ & 8 & 105 & 13.13 & 10.38 & 28 & 1 & 99 & 94.29 & 6 & 5.71 \\
\hline
\end{tabular}

*Número de veces que el curso se apertura.

Fuente: Elaboración propia

\section{Determinación del índice de deserción}

En la tabla 7, por otro lado, se muestra la tasa deserción escolar encontrada en la población y periodo de estudio. 


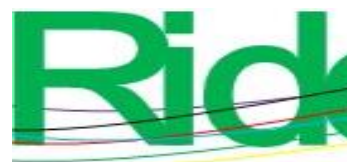

Revista Iberoamericana para la Investigación y el Desarrollo Educativo

ISSN $2007-7467$

Tabla 7. Comportamiento de la deserción escolar en el PELN

\begin{tabular}{|c|c|c|c|c|c|c|c|c|c|}
\hline \multirow[t]{3}{*}{ Generación } & \multicolumn{2}{|c|}{ Ingreso } & \multicolumn{7}{|c|}{ Deserción } \\
\hline & \multirow[t]{2}{*}{$\mathrm{N}$} & \multirow[t]{2}{*}{$\%$} & \multirow[t]{2}{*}{$\mathrm{n}$} & \multirow[t]{2}{*}{$\%$ Gen } & \multirow[t]{2}{*}{$\%$ Total } & \multicolumn{2}{|c|}{ Hombres } & \multicolumn{2}{|c|}{ Mujeres } \\
\hline & & & & & & $\mathrm{n}$ & $\%$ & $\mathrm{n}$ & $\%$ \\
\hline 2010 & 57 & 14.58 & 22 & $39 \%$ & 21.15 & 5 & 5 & 17 & 16.35 \\
\hline 2011 & 58 & 14.83 & 22 & $38 \%$ & 21.15 & 6 & 6 & 16 & 15.38 \\
\hline 2012 & 58 & 14.83 & 23 & $40 \%$ & 22.12 & 1 & 1 & 22 & 21.15 \\
\hline 2013 & 41 & 10.49 & 7 & $17 \%$ & 6.73 & 2 & 2 & 5 & 4.81 \\
\hline 2014 & 52 & 13.30 & 10 & $19 \%$ & 9.62 & 2 & 2 & 8 & 7.69 \\
\hline 2015 & 54 & 13.81 & 11 & $20 \%$ & 10.58 & 1 & 1 & 10 & 9.62 \\
\hline 2016 & 33 & 8.44 & 7 & $21 \%$ & 6.73 & 1 & 1 & 6 & 5.77 \\
\hline 2017 & 38 & 9.72 & 2 & $5 \%$ & 1.92 & 0 & 0 & 2 & 1.92 \\
\hline Totales & 391 & 100 & 104 & $27 \%$ & 100.00 & 18 & 17.31 & 86 & 82.69 \\
\hline
\end{tabular}

Fuente: Elaboración propia

En la tabla 8 se muestra el comportamiento de los ciclos promedios que avanza un desertor y promedio de cursos aprobado y reprobados: una media de $2.35 \pm 0.98$ ciclos avanzados, una media de $8.6 \pm 3.15$ cursos aprobados y una media $6.46 \pm 2.74$ de cursos reprobados al momento de desertar. 


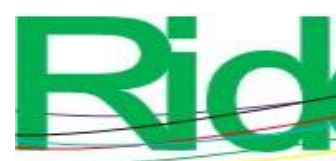

Revista Iberoamericana para la Investigación y el Desarrollo Educativo

ISSN $2007-7467$

Tabla 8. Ciclos promedios avanzados y promedio de cursos aprobado y desaprobados al momento de desertar

\begin{tabular}{|c|c|c|c|c|c|c|c|c|c|c|c|c|}
\hline \multirow{2}{*}{$\begin{array}{l}\text { Generaci } \\
\text { ón }\end{array}$} & \multicolumn{4}{|c|}{ Ciclos avanzados } & \multicolumn{4}{|c|}{ Aprobación de cursos } & \multicolumn{4}{|c|}{ Reprobación de cursos } \\
\hline & $\overline{\mathrm{X}}$ & \pm & $\begin{array}{l}\text { V. } \\
\mathrm{Ma} \\
\mathrm{x} .\end{array}$ & $\begin{array}{l}\mathrm{V} . \\
\mathrm{Mi} \\
\mathrm{n} .\end{array}$ & $\overline{\mathrm{X}}$ & \pm & $\begin{array}{l}\text { V. } \\
\text { Max. }\end{array}$ & $\begin{array}{l}\text { V. } \\
\text { Min. }\end{array}$ & $\overline{\mathrm{X}}$ & \pm & $\begin{array}{l}\text { V. } \\
\text { Max. }\end{array}$ & $\begin{array}{l}\text { V. } \\
\text { Min. }\end{array}$ \\
\hline 2010 & $\begin{array}{r}2.5 \\
9\end{array}$ & $\begin{array}{r}2.0 \\
6\end{array}$ & 10 & 0 & 8 & 7.49 & 32 & 0 & 7.04 & 5.94 & 23 & 0 \\
\hline 2011 & $\begin{array}{r}2.1 \\
8\end{array}$ & 1.5 & 7 & 1 & 7 & 5.95 & 19 & 0 & 5.5 & 3.91 & 14 & 0 \\
\hline 2012 & $\begin{array}{r}2.9 \\
5\end{array}$ & $\begin{array}{r}1.7 \\
7\end{array}$ & 7 & 1 & $\begin{array}{l}1 \\
0\end{array}$ & 6.34 & 20 & 0 & 8.47 & 7.4 & 21 & 0 \\
\hline 2013 & $\begin{array}{r}4.4 \\
2\end{array}$ & 3.4 & 8 & 1 & $\begin{array}{l}1 \\
5\end{array}$ & $\begin{array}{r}11.8 \\
7\end{array}$ & 36 & 2 & $\begin{array}{r}11.5 \\
7\end{array}$ & $\begin{array}{r}11.3 \\
7\end{array}$ & 28 & 1 \\
\hline 2014 & 2.2 & $\begin{array}{r}1.0 \\
3\end{array}$ & 4 & 1 & $\begin{array}{l}1 \\
1\end{array}$ & 8.96 & 25 & 0 & 3.6 & 3.53 & 10 & 0 \\
\hline 2015 & $\begin{array}{r}2.1 \\
8\end{array}$ & $\begin{array}{r}1.6 \\
6\end{array}$ & 5 & 1 & 8 & 5.57 & 22 & 0 & 8.36 & 4.9 & 16 & 2 \\
\hline 2016 & $\begin{array}{r}1.2 \\
8\end{array}$ & $\begin{array}{r}0.4 \\
8\end{array}$ & 2 & 1 & 6 & 4.44 & 12 & 0 & 3.14 & 2.67 & 7 & 0 \\
\hline 2017 & 1 & 0 & 1 & 1 & 4 & 4.24 & 7 & 1 & 4 & 2.82 & 6 & 2 \\
\hline
\end{tabular}

Fuente: Elaboración propia

\section{Relación del índice de reprobación y la deserción escolar}

En la figura 1 se observa la relación entre el índice de desertores por cohorte generacional (2010 a 2017) y el índice de reprobación por cohorte generacional (2010 a 2017) en estudiantes del PELN a través del coeficiente de correlación de Pearson. Como resultado se obtuvo un valor de 0.939 y un valor de $p=0.001$. 

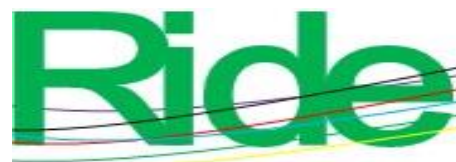

Revista Iberoamericana para la Investigación y el Desarrollo Educativo ISSN $2007-7467$

Figura 1. Relación del porcentaje de desertores con el porcentaje del índice de reprobación

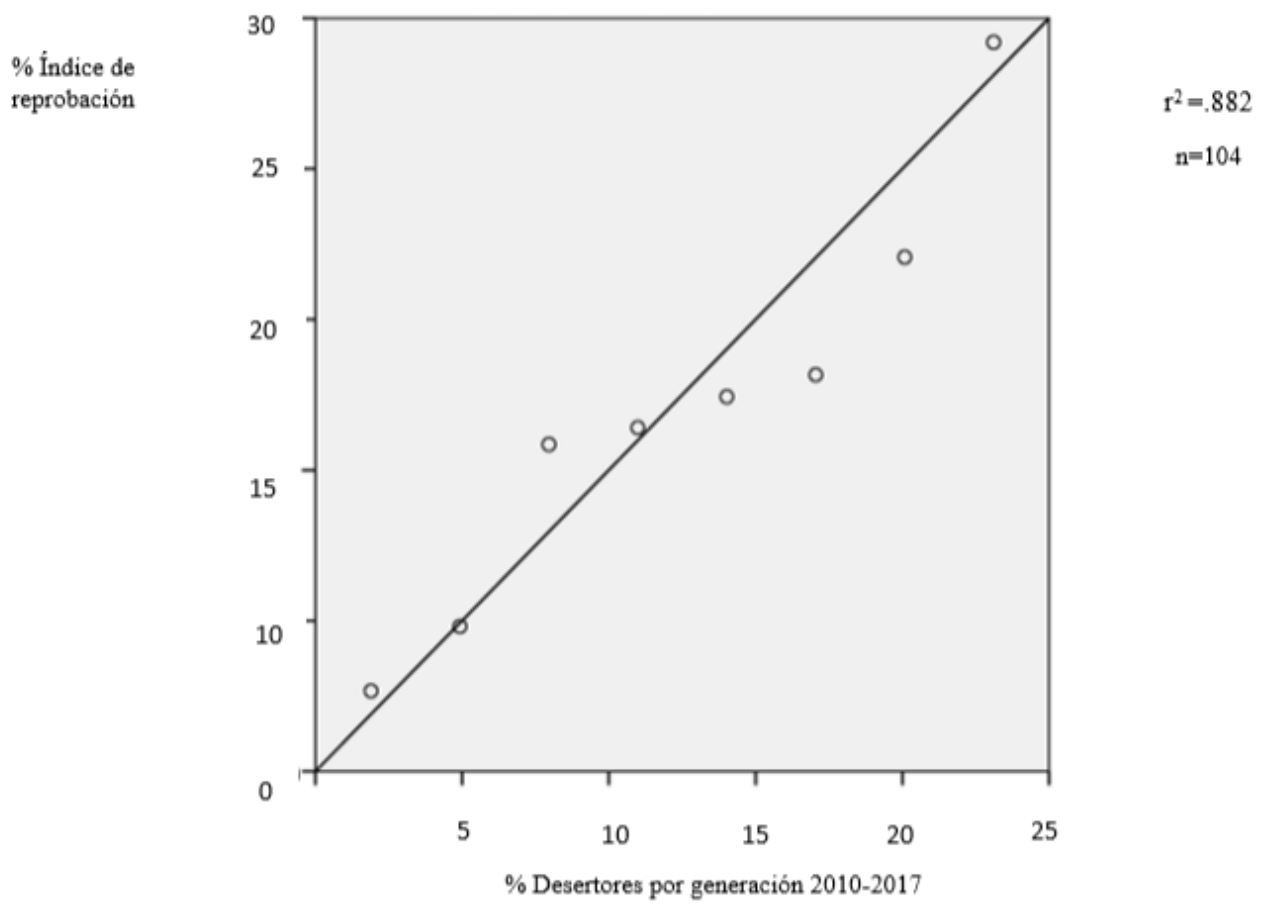

Fuente: SUCE (2019)

\section{Discusión}

Las instituciones de educación superior comúnmente se enfrentan a los fenómenos de reprobación y deserción con un aumento sostenido, lo que ha generado la necesidad de realizar estudios para identificar y atender las causas que los propician; por tanto, las este tipo organizaciones observan con un grado de normalidad estos valores. Una institución educativa en la que no haya reprobados puede provocar sorpresa, incomprensión e indignación. Tradicionalmente se consideraban aceptables tasas de reprobación elevadas, predominantemente en los primeros años de escuela (Ciro y Reyes, 2017). En la actualidad, sin embargo, los organismos evaluadores y acreditadores de programas educativos en México recomiendan disminuir los índices de reprobación a valores menores de 10 \% (Ciees, 2016; Concapren, 2016), debido a que la reprobación es un tema que hay que atender, pues se relaciona con un problema mayor, la deserción escolar.

El PELN cuenta con un plan curricular flexible, que consta de nueve ciclos en los cuales están distribuidos los 52 cursos con 4610 horas totales, de las cuales 2048 son de tipo teóricas, 1906 prácticas y 656 horas de estudio independiente, que se traducen en un total de 301 créditos. 


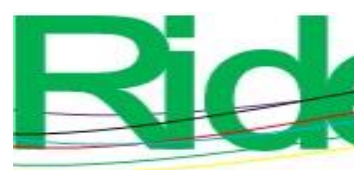

Revista Iberoamericana para la Investigación y el Desarrollo Educativo

ISSN $2007-7467$

De los 52 cursos, $17.31 \%$ (nueve) pertenecen al bloque de las competencias genéricas, $13.46 \%$ (siete) a las competencias interdisciplinarias, $59.62 \%$ (31) a las competencias específicas, más $5.77 \%$ (tres) de cursos que son extracurriculares obligatorios (Nivelación de inglés A y B; Taller de comunicación oral y escrita), y las actividades de formación integral que el estudiante puede ir cubriendo desde el primer ciclo hasta alcanzar las 96 horas, como parte de las competencias genéricas.

\section{Determinación del índice de reprobación}

Existe poca literatura científica con análisis y caracterización de la reprobación por un periodo de siete años, tomando en cuenta cada curso impartido por ciclo escolar. Generalmente solo se determina el porcentaje de reprobación de una cohorte generacional o se aplican encuestas para reconocer las causas de este fenómeno, por lo tanto, no se encontraron puntos de corte con los cuales comparar los índices de reprobación de este estudio. En consecuencia, se determinó tomar como referencia la recomendación emitida por el Concapren (2016) en el informe de acreditación y los resultados del análisis de las academias y el grupo disciplinar "Nutrición, Educación y Administración" (2016) del PELN de la Unacar.

El Concapren (2016) recomienda que el índice de reprobación debe ser menor a $10 \%$, es decir, 9 de cada 10 estudiantes matriculados en un curso deberían aprobar, mientras las academias y el grupo disciplinar del PELN de la universidad ya mencionada (2016) indican que es aceptable un índice de reprobación igual o menor a $30 \%$, de acuerdo con la constante manifestada a partir de los análisis de trayectorias académicas institucionales.

El índice de reprobación general determinado en el PELN es superior a lo indicado por el Concapren (2016), pero menor a lo indicado por el PELN. Al compararlo con otros estudios, se encontró que es similar a lo reportado por Gándara (2014), con $24 \% \pm 4$, pero inferior a lo encontrado por Guzmán (2013), a saber, 47 \%. Cabe señalar que ninguno de los estudios indica el número de veces que se ofrecieron los cursos.

El índice de reprobación de los cursos genéricos muestra que solo $8.33 \%$ de los cursos manifiestan menos de $10 \%$ de reprobación, como lo sugiere Concapren (2016), pero según el criterio de las academias del PELN, $33.33 \%$ de los cursos tienen un índice de reprobación aceptable. Analizando las veces promedio en que se aperturaron los cursos en las competencias genéricas, se puede observar que la media de reprobación se incrementa al doble, $51 \%$, es decir, en siete ocasiones que se debieron ofrecer los cursos, y se apertura el doble de veces, 14 veces, lo

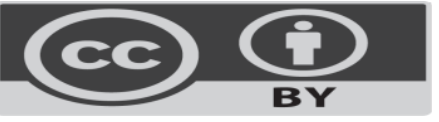




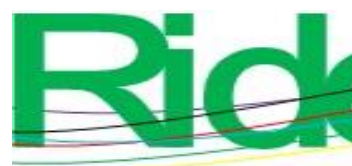

Revista Iberoamericana para la Investigación y el Desarrollo Educativo

ISSN $2007-7467$

cual enmascara el problema, y la solución no es el número de veces que se ofrezca un curso para disminuir la reprobación; sino las estrategias que se deben generar para disminuir la reprobación dentro del número de veces de acuerdo con el plan estratégico de la trayectoria escolar por cohorte generacional.

En relación con el índice de reprobación de los cursos que contribuyen a las competencias interdisciplinarias, se identifica que ningún curso cumple con el criterio de los organismos nacionales, mientras que $42 \%$ de estos cumple el criterio del PELN, con lo que se determina que el número de veces de un mismo curso ofertado sigue siendo un factor que continúa enmascarando el fenómeno de la reprobación, ya que se identificó que la media de apertura fue el doble de veces, reciclando estudiantes reprobados, es decir, que el índice de reprobación de estos cursos es de 62 $\%$ y no de $31 \%$.

En el análisis de los cursos de las competencias específicas se identificó que el índice de reprobación de estos es menor a los encontrados en los de las competencias genéricas e interdisciplinarias, con una media de apertura de cursos correspondiente al plan estratégicos de trayectoria escolar de las cohortes generacionales, donde el factor de veces que se ofreció un mismo curso no afectó el índice de reprobación.

Hay que aclarar que la administración de los cursos de las competencias genéricas e interdisciplinarias no depende de la gestoría del PELN, como en el caso de los cursos de las específicas, lo cual pone de manifiesto la limitada injerencia que tienen los actores del programa educativo sobre los cursos de las primeras dos competencias aquí mencionadas, ya que su participación se limita a la solicitud de los cursos que se ofertarán en el periodo escolar correspondiente.

Dentro de los cursos que conforman las competencias específicas, a saber, los disciplinares del PELN: básicos, profesionalizantes, optativos y terminales, el índice de reprobación estuvo en promedio dentro de lo propuesto por los organismos reguladores y las academias del programa educativo, así como el número de veces de apertura de un mismo curso se mantuvo dentro del plan estratégico por cohorte generacional, lo que indica que el ejercicio estratégico efectuado dentro de estos debería aplicarse en los cursos de las competencias genéricas e interdisciplinares para lograr un equilibrio y una continuidad en el desempeño académico de los estudiantes. 


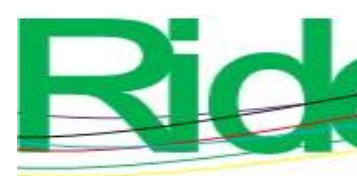

Revista Iberoamericana para la

Investigación y el Desarrollo Educativo

ISSN $2007-7467$

\section{Determinación del índice de deserción}

La deserción de los estudiantes universitarios es un tema controversial dentro de las instituciones de educación superior. Y como ya se mencionó, las causas son multifactoriales (Torres et al., 2017). La tasa de deserción escolar encontrada en este estudio es menor a lo indicado por la Organización para la Cooperación y el Desarrollo Económicos [OCDE] (2016) para el caso de México, con $50 \%$, y por Torres et al. (2017), $44 \%$, pero similar a lo reportado por Piratoba y Barbosa (2013): $28.75 \%$.

Hace algunos años, Torres et al. (2017) identificaron los factores que afectan este fenómeno en el PELN. Fueron seis factores predominantes: 1) $47 \%$ por cambio de programa educativo, 2) $16 \%$ por cambio de carrera e institución educativa local, 3) $12 \%$ cambio a la misma carrera, pero en una institución foránea, generalmente por cambio de residencia, 4) $12 \%$ por problemas familiares, 5) $8 \%$ por problemas de salud y 6) $5 \%$ por situación económica. Estos datos fueron obtenidos de las cédulas de baja institucional, donde el alumno indica los motivos de baja definitiva; sin embargo, no se realizó un análisis de trayectoria escolar, específicamente el fenómeno de la reprobación, que es algo que no indican los estudiantes, pues tienden a excusar su deserción.

En esa tónica, $96 \%$ de los estudiantes desertores en sus trayectorias escolares tienen entre $40 \%$ y $80 \%$ de cursos reprobados. Un fenómeno identificado en el presente estudio fue el comportamiento de un estudiante desertor con respecto a los ciclos promedios que avanza y el promedio de cursos aprobados y reprobados; se encontró una media de $2.35 \pm 0.98$ ciclos avanzados, una media de $8.6 \pm 3.15$ cursos aprobados y una media $6.46 \pm 2.74$ de cursos reprobados al momento de desertar.

\section{Relación del índice de reprobación y la deserción escolar}

En el presente estudio se determina que sí existe una fuerte relación entre la reprobación y la deserción escolar, tal y como se muestra en el modelo de regresión lineal (figura 1), en el cual se obtuvo un valor de $r^{2}=0.882$. 


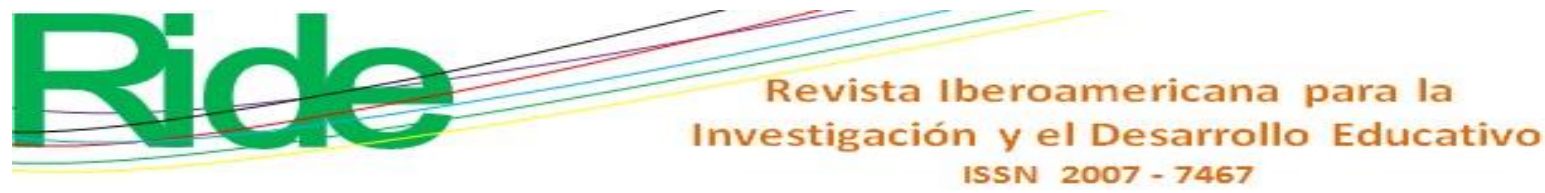

\section{Conclusiones}

En los últimos años, han incrementado los estudios sobre la reprobación, deserción, suspensión de estudios $\mathrm{y}$, en general, lo relacionado con el éxito o fracaso escolar. Esta investigación permitió identificar que existe una relación positiva entre el número de desertores por generación y el índice de reprobación, es decir, entre mayor número de cursos no aprobados, mayor posibilidad de deserción existe.

El índice de reprobación general no manifiesta ser un problema grave para el PELN, pero, al analizar a profundidad este fenómeno, se identificó que los cursos de las competencias genéricas e interdisciplinares son los que tienen el mayor impacto sobre la deserción,

El número de veces que se apertura un curso enmascara el problema, por lo tanto, se debe actuar sobre este factor, desde la propuesta de los grupos en el sistema, ya que genera pérdidas económicas para la institución y problemas en las trayectorias académicas de los estudiantes.

El tiempo promedio que avanza un desertor es de un año y medio, es decir, tres ciclos aproximadamente. Durante esa trayectoria escolar se observa un promedio de tres cursos aprobados y una media de seis cursos reprobados al momento de desertar.

Esta investigación robustece los resultados presentados por otros autores. Además, aporta información novedosa para el diseño de estrategias de intervención que contribuyan a erradicar el fenómeno de la reprobación y, de esta manera, al éxito académico de los alumnos universitarios.

Se recomienda realizar un estudio sobre las causas de reprobación desde la perspectiva del estudiante para poder desarrollar estrategias de intervención oportunas y, por ende, atender y disminuir este fenómeno institucional.

\section{Agradecimientos}

Agradecemos a la dirección de posgrado por el financiamiento del proyecto Sucesos estresantes en los estudiantes de la Universidad Autónoma del Carmen. Un diagnóstico necesario para fortalecer el sistema de tutoría, así como al Grupo Interdisciplinario de Investigación de las Violencias en el Sureste de México A. C. (Giiviss) por la facilitación de bases de datos que fueron de gran apoyo para la realización de este producto, y a la dirección de la FCS por todas las facilidades brindadas. 


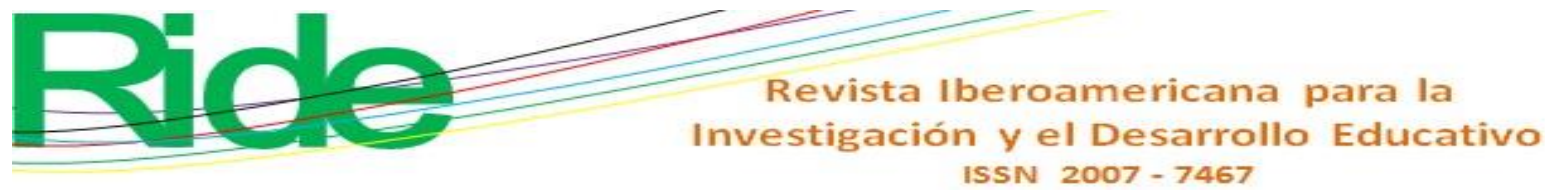

\section{Referencias}

Castellanos, M. C., Alvarado, L. D. and Villamil, J. E. (2018). University Student Desertion Analysis using Agent-Based Modeling Approach. In Proceedings of the 3rd International Conference on Complexity, Future Information Systems and Risk - Volume 1 (pp. 128-135). SciTePress.

Ciro, S. y Reyes, C. M. (2017). Principales causas de reprobación de alumnos de los grupos de quinto semestre grupo seis y ocho de la escuela preparatoria número tres. (Capítulo I Antecedentes). Con-Ciencia Boletín Científico de la Escuela Preparatoria No. 3, 4(7). Recuperado de https://repository.uaeh.edu.mx/revistas/index.php/prepa3/article/view/2079. Comités Interinstitucionales para la Evaluación de la Educación Superior [Ciees]. (2016). Informe de Evaluación del programa de Licenciatura en Nutrición de la Universidad Autónoma del Carmen. Ciudad de México, México: Comités Interinstitucionales para la Evaluación de la Educación Superior.

Consejo Nacional para la Calidad de Programas Educativos en Nutriología [Concapren]. (2016). Informe del proceso de acreditación del programa de Licenciatura en Nutrición de la Universidad Autónoma del Carmen (001). Ciudad de México, México: Consejo Nacional para la Calidad de Programas Educativos en Nutriología.

Fallis, R. K. and Opotow, S. (2003). Are Students Failing School or Are Schools Failing Students? Class Cutting in High School. Journal of Social Issues, 59(1), 103-119.

Flores, S., Camacho, A. y Ontiveros, R. (2013). Análisis estadístico de las causas de reprobación desde la perspectiva del alumno de ingeniería del Instituto Tecnológico de Chihuahua II. Recuperado de http://www.chi.itesm.mx/investigacion/wpcontent/uploads/2013/11/EDU42.pdf.

Gándara, F. J. (2014). Herramientas de calidad y el trabajo en equipo para disminuir la reprobación escolar. Conciencia Tecnológica, (48), 17-24. Recuperado de https://www.redalyc.org/pdf/944/94432996003.pdf

Guzmán, C. H. (2013). Reprobación y Desinterés en Alumnos de Ingeniería Mecatrónica. Orbis. Revista Científica Ciencias Humanas, 9(25), 33-46. Recuperado de https://www.redalyc.org/pdf/709/70928419003.pdf.

Mendoza, D. C. (2016). Dimensión de la problemática sobre el bajo rendimiento, la reprobación y la deserción de los jóvenes bachilleres. En Reprobación y deserción en el bachillerato. Elementos para el análisis de la equidad y la eficacia escolar (pp. 78-86). México: 


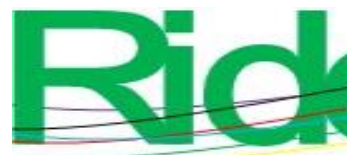

Universidad
Revista Iberoamericana para la Investigación y el Desarrollo Educativo ISSN $2007-7467$

Recuperado

https://ibero.mx/sites/all/themes/ibero/descargables/publicaciones/reprobacion-ydesercion.pdf?_ga=2.188307489.821269960.1496937738-467588176.1494006552.

Organización para la Cooperación y Desarrollo Económico [OCDE]. (2016). Panorama de la sociedad 2016. Un primer plano sobre los jóvenes. La situación de México. México: Organización para la Cooperación y Desarrollo Económico. Recuperado de https://www.oecd.org/mexico/sag2016-mexico.pdf.

Piratoba, B. N. y Barbosa, O. O. (2013). Factores de deserción de los estudiantes en la facultad de enfermería de la Universidad de Ciencias Aplicadas y Ambientales U.D.C.A, durante el periodo: 2009-2010-I 2011. Revista U.D.C.A Actualidad \& Divulgación Científica, 16(2), 553-562.

Recuperado

de

http://www.scielo.org.co/scielo.php?script=sci_arttext\&pid=S0123$42262013000200031 \& \operatorname{lng}=$ en $\&$ tlng=es.

Sánchez, S. A. (2015). La reprobación, principal factor que origina la deserción escolar en la educación media superior en León, Guanajuato. REAXION Ciencia y Tecnología Universitaria, $2(3)$, 29-24. Recuperado de http://reaxion.utleon.edu.mx/Art_La_reprobacion_principal_factor_que_origina_la_deserci on_escolar_en_la_educacion_media_superior_en_Leon_Guanajuato.html.

Secretaría de Educación Pública [SEP]-Consejo para la Evaluación de la Educación del Tipo Medio Superior [Copeems]. (2012). Reporte de la Encuesta Nacional de la Deserción en la Educación Media Superior. México: Secretaría de Educación Pública-Consejo para la Evaluación de la Educación del Tipo Medio Superior.

Sistema Universitario de Control Escolar [SUCE]. (2019). Datos de aprobación y reprobación de cursos y deserción del programa educativo de licenciatura en Nutrición (PELN). Campeche, México: Universidad Autónoma del Carmen.

Torres, A. E., Acuña, J. P., Guadarrama, C., Solís, O. C. y Flores, P. (2017). Deserción Universitaria. Una aproximación a las características de abandono escolar en estudiantes de la Licenciatura en Nutrición para la toma de decisiones. Transformación (Con)ciencia Educativa. Nuestras Voces. Nuestras Acciones, 2(4), 12-20. Recuperado de http://transformacion.setab.gob.mx/index.php/es/usingjoomla/extensions/components/content-component/article-categories/87-indices/88-indiceano-2-numero-4. 


\begin{tabular}{|l|l|}
\hline Rol de Contribución & Autor (es) \\
\hline Conceptualización & Ángel Esteban Torres-Zapata \\
\hline Metodología & Javier Rivera Domínguez \\
\hline Software & Patricia Flores López \\
\hline Validación & María del Pilar García Reyes \\
\hline Análisis Formal & Ángel Esteban Torres-Zapata \\
\hline Investigación & Ángel Esteban Torres-Zapata \\
\hline Recursos & Dariola Astrid Castillo Trejo \\
\hline Curación de datos & María del Pilar García Reyes \\
\hline $\begin{array}{l}\text { Escritura - Preparación del } \\
\text { borrador original }\end{array}$ & Javier Rivera Domínguez \\
\hline $\begin{array}{l}\text { Escritura - Revisión y y } \\
\text { edición }\end{array}$ & Ángel Esteban Torres-Zapata \\
\hline Visualización & Dariola Astrid Castillo Trejo \\
\hline Supervisión & Ángel Esteban Torres-Zapata \\
\hline $\begin{array}{l}\text { Administración } \\
\text { Proyectos }\end{array}$ & Ángel Esteban Torres-Zapata \\
\hline Adquisición de fondos & Dariola Astrid Castillo Trejo \\
\hline
\end{tabular}

